\title{
Theoretical Prediction and Experimental Measurement of Embedded Optical Fiber Strain Sensors
}

\author{
Shiuh-Chuan Her* and Chih-Ying Huang \\ Department of Mechanical Engineering, Yuan Ze University, \\ 135 Yuan-Tung Road, Chungli, Taoyuan, Taiwan, R.O.C.
}

(Received December 24, 2013; accepted March 6, 2014)

Key words: optical fiber strain sensor, Mach-Zehnder interferometer, strain transformation, embedded-fiber length

A theoretical prediction of the strain transferred from a host material to an embedded optical fiber was presented to reveal the differential strain between the optical fiber sensor and host material. Experiments using the Mach-Zehnder interferometric-type fiber-optic sensor were conducted to measure the strain in the embedded optical fiber sensor induced by the host structure. Experimental results show that the strain measured in the optical fiber is less than the true strain in the host structure. The percentage of strain in the host structure which actually transferred to the optical fiber is dependent on the length of the embedded optical fiber. The general trend of the strain transformation obtained from the experiments shows that the longer the embedded optical fiber, the greater the strain that is transferred to the optical fiber.

\section{Introduction}

Optical fiber sensors have been widely adapted as primary sensing devices for monitoring the strain and temperature of structures under operating conditions. As the optical fiber is extremely small, it can be embedded into structures for determining the strain distribution without affecting the mechanical properties of the host structures. Schizas and Karalekas ${ }^{(1)}$ used fiber Bragg grating sensors (FBGs) to monitor the strain development during solidification of a photocurable resin. Wan and Leung ${ }^{(2)}$ developed an optical fiber sensor for monitoring mixed-mode cracks in structures. Yuan et al. (3) $^{(3)}$ designed a twin multiplexing strain sensing array based on a low-coherence fiber optic Mach-Zehnder interferometer. Most of the results from these research studies are based on the assumption that the optical fiber sensors are perfectly bonded on the host structure, so that the strain from the host structure will be transferred completely to the optical fiber. However, owing to the existence of the adhesive layer and protective coating,

${ }^{*}$ Corresponding author: e-mail: mesch@saturn.yzu.edu.tw 
part of the energy would convert to shear deformation. ${ }^{(4)}$ The strain measured using an embedded optical fiber is highly dependent on the bonding characteristics of the adhesive layer and protective coating as well as the bonded-fiber length. Cheng et al. ${ }^{(5)}$ showed that the strain transferred from the substrate to the bonded FBG depends on the shear lag parameters, the bonded-fiber length and the stiffness ratio between the substrate and the FBG. Li et al. ${ }^{(6)}$ proposed an analytical model to characterize the strain transmission of surface-bonded FBG sensors. Her and Tsai ${ }^{(7)}$ evaluated the strain in optical fiber sensors induced by the host structure. Optical fibers can be either embedded in the host structure during the fabrication or bonded on the surface of the structure by way of adherents. For a sensor, it is expected that the strains between the optical fiber and host structure are the same. However, owing to the existence of the adhesive layer and protective coating, the strain in the optical fiber is less than that in the host structure. In this investigation, a theoretical prediction of the strain transferred from the host material to the embedded optical fiber is presented to reveal the differential strain between the optical fiber sensor and the host material. The theoretical predictions are validated with experiments using a Mach-Zehnder interferometric-type fiber-optic sensor. Good agreement between the theoretical and experimental results shows that the proposed approach can provide an efficient method for calculating the strain in an optical fiber.

\section{Strain Analysis of Embedded Optical Fiber Sensor}

The analytical model is shown in Fig. 1 with three concentric cylinders representing the optical fiber, protective coating and host material. The transformation of the strain between the host structure and embedded optical fiber sensor was derived on the

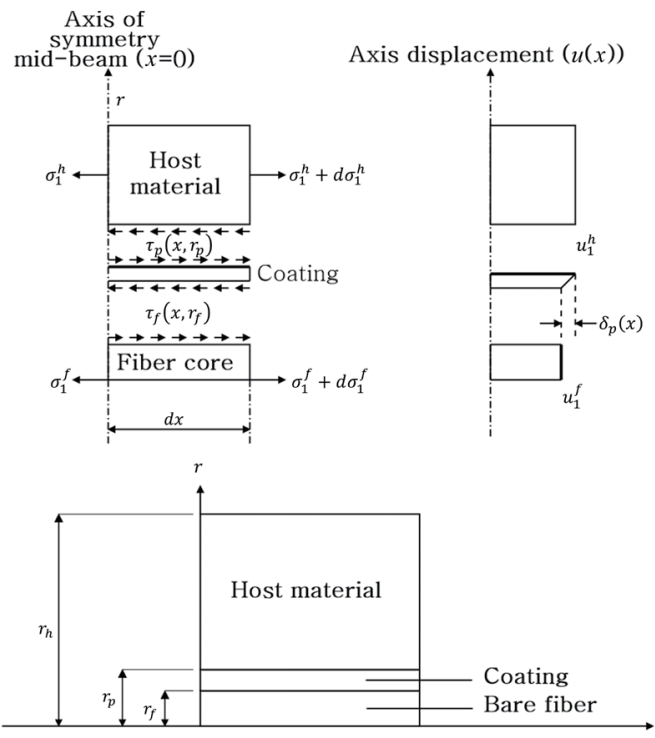

Fig. 1. Analytical model of embedded optical fiber. 
basis of the following assumptions: (1) all interfaces are perfectly bonded, i.e., there is displacement continuity along interfaces and (2) the protective coating is subjected to shear deformation only. This assumption is reasonable since the Young's modulus of the coating is significantly smaller than those of the host structure and optical fiber. Recently, Her and Tsai ${ }^{(7)}$ have developed a theoretical model to predict the strain in an embedded optical fiber sensor induced by the host material as

$$
\begin{gathered}
\varepsilon_{1}^{\mathrm{f}}(x)=\frac{E_{\mathrm{h}}\left(r_{\mathrm{h}}^{2}-r_{\mathrm{p}}^{2}\right) \varepsilon_{0}}{E_{\mathrm{f}} r_{\mathrm{f}}^{2}+E_{\mathrm{h}}\left(r_{\mathrm{h}}^{2}-r_{\mathrm{p}}^{2}\right)}\left[1-\frac{\cosh \left(\lambda_{1} x\right)}{\cosh \left(\lambda_{1} L_{\mathrm{f}}\right)}\right], \\
\lambda_{1}=\sqrt{\frac{2 G_{\mathrm{p}}}{r_{\mathrm{f}}^{2} \ln \left(r_{\mathrm{p}} / r_{\mathrm{f}}\right)}\left[\frac{E_{\mathrm{f}} r_{\mathrm{f}}^{2}+E_{\mathrm{h}}\left(r_{\mathrm{h}}^{2}-r_{\mathrm{p}}^{2}\right)}{E_{\mathrm{f}} E_{\mathrm{h}}\left(r_{\mathrm{h}}^{2}-r_{\mathrm{p}}^{2}\right)}\right]} .
\end{gathered}
$$

Here, $E_{\mathrm{h}}$ and $E_{\mathrm{f}}$ are the Young's moduli of the host material and optical fiber, respectively. $G_{\mathrm{p}}$ is the shear modulus of the protective coating; $r_{\mathrm{h}}, r_{\mathrm{p}}$, and $r_{\mathrm{f}}$ are the radii of the host material, protective coating and optical fiber, respectively; $L_{\mathrm{f}}$ is half of the embeddedfiber length; $\varepsilon_{0}$ is the strain applied to the host material.

\section{Mach-Zehnder Interferometer}

The schematic diagram of a Mach-Zehnder interferometer is shown in Fig. 2. It consists of two $2 \times 2$ couplers, one each at the input and output. Excitation is applied to the sensing fiber, resulting in an optical path difference between the reference and sensing fibers. The light intensity at the output of the Mach-Zehnder interferometer can be expressed as ${ }^{(8)}$

$$
\begin{gathered}
I=2 \mathrm{~A}^{2}(1+\cos \Delta \varphi) \\
\Delta \varphi=\frac{2 \pi n_{0}}{\lambda}\left\{1-\frac{n_{0}}{2}\left[(1-v) p_{12}-v p_{11}\right]\right\} \int_{\Gamma} e_{1}^{\mathrm{f}} \mathrm{d} x,
\end{gathered}
$$

where $\Delta \varphi$ is the optical phase shift, $n_{0}$ is the refractive index of the optical fiber, $\lambda$ is the optical wavelength, $v$ is Poisson's ratio, $p_{11}$ and $p_{12}$ are Pockel's constants, and $\varepsilon_{1}^{\mathrm{f}}$ is the strain in the optical fiber. Since the terms in front of the integral sign of eq. (2) are constants for any given optical fiber system, the total optical phase shift $\Delta \varphi$ is

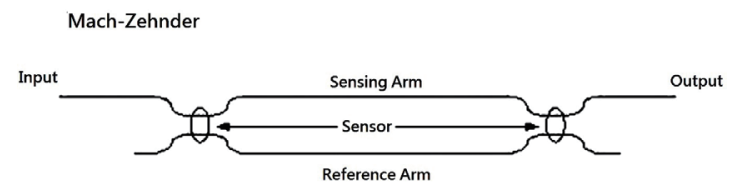

Fig. 2. Mach-Zehnder interferometer. 
proportional to the integral of the optical fiber strain. By measuring the total optical phase shift, the integral of the optical fiber strain can be easily obtained as

$$
\int_{\Gamma_{s}} e_{1}^{\mathrm{f}} \mathrm{d} x=\frac{\Delta \varphi}{\frac{2 \pi n_{0}}{\lambda}\left\{1-\frac{1}{2} n_{0}^{2}\left[\left(1-v_{\mathrm{f}}\right) p_{12}-v_{\mathrm{f}} p_{11}\right]\right\}} .
$$

The light intensity shown in eq. (2) is a cosine function of the optical phase shift $\Delta \varphi$. Thus, the light intensity of the Mach-Zehnder interferometer exhibits a periodic change with the optical phase shift $\Delta \varphi$. As the optical phase shift $\Delta \varphi=2 \pi$, the light intensity goes through a complete cycle. The corresponding integral of the strain is

$$
\begin{aligned}
\Delta s & =\int_{\Gamma_{s}} \varepsilon_{1}^{\mathrm{f}} \mathrm{d} x=\frac{2 \pi}{\frac{2 \pi n_{0}}{\lambda}\left\{1-\frac{1}{2} n_{0}^{2}\left[\left(1-v_{\mathrm{f}}\right) p_{12}-v_{\mathrm{f}} p_{11}\right]\right\}} \\
& =\frac{\lambda}{n_{0}\left\{1-\frac{1}{2} n_{0}^{2}\left[\left(1-v_{\mathrm{f}}\right) p_{12}-v_{\mathrm{f}} p_{11}\right]\right\}} .
\end{aligned}
$$

The integral of the strain in eq. (4) denotes the change in the length of the sensing fiber, which is embedded in the host material. The average strain in the embedded optical fiber for optical phase shift $\Delta \phi=2 \pi$ is

$$
\Delta \varepsilon_{\text {avg }}=\frac{\Delta s}{s}=\frac{\int_{\Gamma_{s}} \varepsilon_{\mathrm{f}} \mathrm{d} x}{2 L_{\mathrm{f}}}=\frac{\lambda}{2 L_{\mathrm{f}} n_{0}\left\{1-\frac{1}{2} n_{0}^{2}\left[\left(1-v_{\mathrm{f}}\right) p_{12}-v_{\mathrm{f}} p_{11}\right]\right\}} .
$$

Equation (5) represents the average strain in the optical fiber as the light intensity performs one complete cycle. The total average strain in the embedded optical fiber can be determined by counting the number of cycles of interferometric light intensity as follows.

$$
\left(\varepsilon_{1}^{\mathrm{f}}\right)_{\exp }=m \Delta \varepsilon_{\mathrm{avg}}=\frac{m \lambda}{2 L_{\mathrm{f}} n_{0}\left\{1-\frac{1}{2} n_{0}^{2}\left[\left(1-v_{\mathrm{f}}\right) p_{12}-v_{\mathrm{f}} p_{11}\right]\right\}}
$$

Here, $m$ is the number of cycles of light intensity determined from the experimental result; it can be an integer or a noninteger. Thus, the experimental measurement of the average strain in the embedded optical fiber by the Mach-Zehnder interferometric technique can be obtained from eq. (6).

The theoretical value of the average strain in the embedded optical fiber can be deduced from eq. (1).

$$
\begin{aligned}
\left(\varepsilon_{1}^{\mathrm{f}}\right)_{\text {the }} & =\frac{\int_{\varepsilon_{1}^{\mathrm{f}}} \mathrm{d} x}{2 L_{\mathrm{f}}}=\frac{2 \int_{0}^{L_{\mathrm{f}}} \frac{E_{\mathrm{h}}\left(r_{\mathrm{h}}^{2}-r_{\mathrm{p}}^{2}\right) \varepsilon_{0}}{E_{\mathrm{f}} r_{\mathrm{f}}^{2}+E_{\mathrm{h}}\left(r_{\mathrm{h}}^{2}-r_{\mathrm{p}}^{2}\right)}\left[1-\frac{\cosh \left(\lambda_{1} x\right)}{\cosh \left(\lambda_{1} L_{\mathrm{f}}\right)}\right] \mathrm{d} x}{2 L_{\mathrm{f}}} \\
= & \frac{E_{\mathrm{h}}\left(r_{\mathrm{h}}^{2}-r_{\mathrm{p}}^{2}\right) \varepsilon_{0}}{E_{\mathrm{f}} r_{\mathrm{f}}^{2}+E_{\mathrm{h}}\left(r_{\mathrm{h}}^{2}-r_{\mathrm{p}}^{2}\right)}\left[1-\frac{\sinh \left(\lambda_{1} L_{\mathrm{f}}\right)}{L_{\mathrm{f}} \lambda_{1} \cosh \left(\lambda_{1} L_{\mathrm{f}}\right)}\right]
\end{aligned}
$$


To evaluate the percentage of strain transferred from the host structure to the embedded optical fiber, the coefficient of strain transformation between the optical fiber and host structure is defined as

$$
\begin{gathered}
(K)_{\exp }=\frac{\left(\varepsilon_{1}^{\mathrm{f}}\right)_{\exp }}{\varepsilon_{0}}=\frac{m \lambda}{2 L_{\mathrm{f}} n_{0}\left\{1-\frac{1}{2} n_{0}^{2}\left[\left(1-v_{\mathrm{f}}\right) p_{12}-v_{\mathrm{f}} p_{11}\right]\right\} \varepsilon_{0}}, \\
(K)_{\text {the }}=\frac{\left(\varepsilon_{1}^{\mathrm{f}}\right)_{\text {the }}}{\varepsilon_{0}}=\frac{E_{\mathrm{h}}\left(r_{\mathrm{h}}^{2}-r_{\mathrm{p}}^{2}\right)}{E_{\mathrm{f}} r_{\mathrm{f}}^{2}+E_{\mathrm{h}}\left(r_{\mathrm{h}}^{2}-r_{\mathrm{p}}^{2}\right)}\left[1-\frac{\sinh \left(\lambda_{1} L_{\mathrm{f}}\right)}{L_{\mathrm{f}} \lambda_{1} \cosh \left(\lambda_{1} L_{\mathrm{f}}\right)}\right] .
\end{gathered}
$$

\section{Experimental Measurements}

A uniaxial tensile test was employed to investigate the strain response measured using an embedded optical fiber sensor. A bare optical fiber is embedded in the specimen as the sensing fiber of the Mach-Zehnder interferometer. A strain gauge is bonded on the top surface of the specimen to measure the far-field strain $\varepsilon_{0}$ of the host material induced by the tensile test. The Young's moduli of the optical fiber, coating and host structure are $72 \mathrm{GPa}, 6.7 \mathrm{MPa}$ and $2 \mathrm{GPa}$, respectively. The outer radii of the optical fiber and coating are $r_{\mathrm{f}}=62.5 \mu \mathrm{m}$ and $r_{\mathrm{p}}=125 \mu \mathrm{m}$, respectively; the thickness of the host structure is $h=5 \mathrm{~mm}$. The index of refraction and Pockel's constants ${ }^{(9)}$ are $n_{0}=1.45, p_{11}$ $=0.12$ and $p_{12}=0.27$, respectively. The wavelength of the light emitted from the laser diode to the optical fiber is $\lambda=1549.2 \mathrm{~nm}$. The length of the embedded optical fiber is varied from $2 L_{\mathrm{f}}=40$ to $120 \mathrm{~mm}$ in increments of $10 \mathrm{~mm}$ to investigate the effect of the embedded-fiber length on the strain measurement. Uniaxial tensile tests were conducted using an Instron material test system. Three tests were performed for each sample. The average of three measured values was taken for each sample. Figure 3 shows the results of the strain in the test specimen, measured using the strain gauge and the light intensity of the Mach-Zehnder interferometer with an embedded-fiber length of $100 \mathrm{~mm}$. Counting the number of cycles of light intensity from Fig. 3 and then substituting into
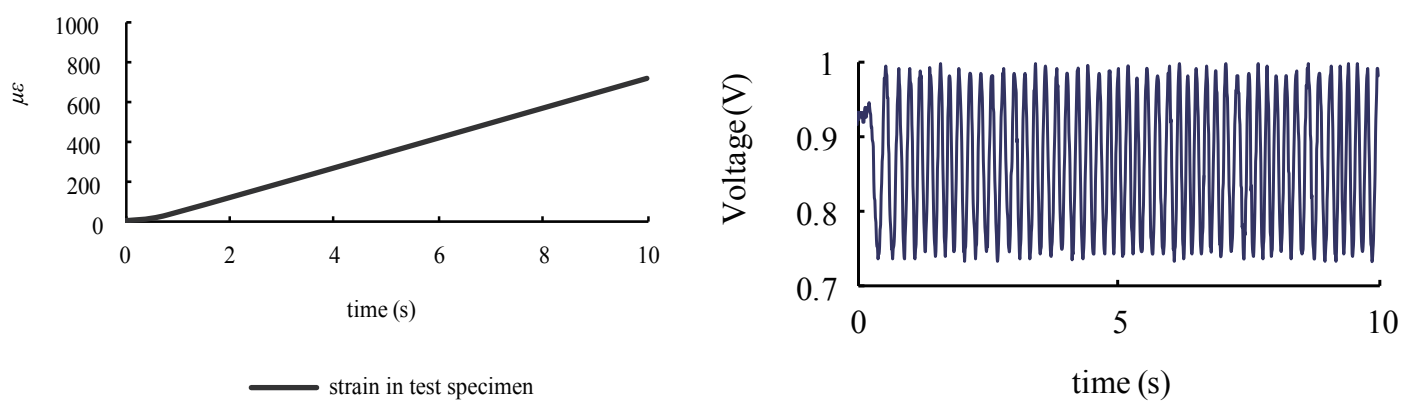

Fig. 3. (Color online) Strain in test specimen measured using strain gauge and light intensity of Mach-Zehnder interferometer with embedded-fiber length of $100 \mathrm{~mm}$. 
eq. (6) yields the experimental result of the average strain in the embedded optical fiber. Theoretical prediction of the average strain in the optical fiber is obtained by substituting the strain in the test specimen measured using the strain gauge into eq. (7). The results of average strain in the embedded optical fiber attained by experimental measurement and theoretical prediction with various embedded-fiber lengths are presented in Table 1. Good agreements are achieved between the results of the theoretical prediction [eq. (7)] and the experimental measurement [eq. (6)]. Experimental measurement and theoretical prediction of the percentage of strain transferred from the host structure to the embedded optical fiber can be obtained using eqs. (8) and (9), respectively. Figure 4 shows the results of the coefficient of strain transformation varying with the embedded-fiber length. It can be observed that the strain in the embedded optical fiber is not the same as that of the host structure. The longer the embedded-fiber length, the larger the coefficient of strain transformation, i.e., the greater the strain that is transferred to the optical fiber.

Table 1

Average strain of optical fiber obtained by experimental measurement [eq. (6)] and theoretical prediction [eq. (7)].

\begin{tabular}{lccccc}
\hline $\begin{array}{l}\text { Embedded- } \\
\text { fiber } \\
\text { length }\left(2 L_{\mathrm{f}}\right)\end{array}$ & $\begin{array}{c}\text { Host material } \\
\text { strain } \varepsilon_{0}(\mu \varepsilon)\end{array}$ & $\begin{array}{c}\text { Number } \\
\text { of cycles }\end{array}$ & $\begin{array}{c}\text { Experimental } \\
\text { measurement [eq. (6)] } \\
\text { of optical fiber strain } \\
\varepsilon_{\mathrm{f}}(\mu \varepsilon)\end{array}$ & $\begin{array}{c}\text { Theoretical prediction } \\
\text { [eq. (7)] of optical } \\
\text { fiber strain } \varepsilon_{\mathrm{f}}(\mu \varepsilon)\end{array}$ & $\begin{array}{c}\text { Difference } \\
(\%)\end{array}$ \\
\hline $40 \mathrm{~mm}$ & 915 & 15.5 & 576 & 625 & 7.84 \\
$50 \mathrm{~mm}$ & 923 & 21.7 & 647 & 684 & 5.41 \\
$60 \mathrm{~mm}$ & 944 & 28.6 & 710 & 751 & 5.46 \\
$70 \mathrm{~mm}$ & 822 & 31.7 & 674 & 647 & 4.17 \\
$80 \mathrm{~mm}$ & 569 & 25.7 & 478 & 502 & 4.78 \\
$90 \mathrm{~mm}$ & 609 & 31.4 & 519 & 532 & 2.44 \\
$100 \mathrm{~mm}$ & 602 & 35.2 & 525 & 502 & 4.58 \\
$110 \mathrm{~mm}$ & 618 & 40.8 & 552 & 548 & 0.73 \\
$120 \mathrm{~mm}$ & 588 & 43.0 & 534 & 515 & 3.69 \\
\hline
\end{tabular}

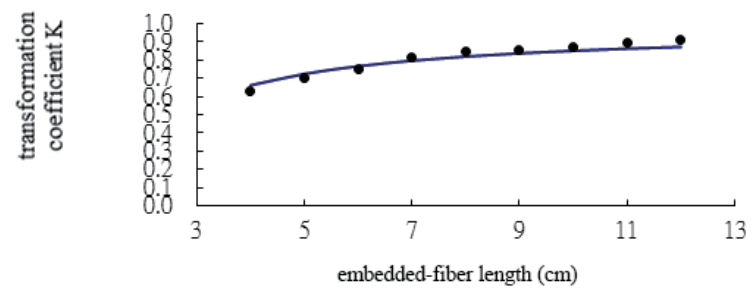

— theoretical prediction $\mathrm{K} \quad$ - experimental measurement $\mathrm{K}$

Fig. 4. (Color online) Variation of strain transformation coefficient $K$ with embedded-fiber length. 


\section{Conclusions}

In this work, the strain in an embedded optical fiber induced by the host structure was presented. The theoretical predictions were validated by the experimental results using a Mach-Zehnder interferometer. Good agreement between them shows that the proposed approach can provide an efficient way for calculation of the strain in an optical fiber. The percentage of the strain in the host material actually transferred to the embedded optical fiber is dependent on the bonding characteristics of the protective coating and host material as well as the embedded-fiber length. Experimental results show that the strain measured in the optical fiber is lower than the true strain in the host structure. The longer the embedded-fiber length, the greater the strain that is transferred to the optical fiber.

\section{Acknowledgements}

The authors are thankful for the financial support by the National Science Council, Taiwan, under grant no. NSC 102-2622-E-155-014-CC3.

\section{References}

1 C. Schizas and D. Karalekas: J. Mater. Process. Technol. 209 (2009) 2349.

2 K. T. Wan and C. K. Y. Leung: Sens. Actuators, A 135 (2007) 370.

3 L. Yuan, Q. Wen, C. Liu, Y. Jie and G. Li: Sens. Actuators, A 135 (2007) 152.

4 K. T. Lau, L. M. Yuan, L. Zhou, J. Wu and C. H. Woo: Compos. Struct. 51 (2001) 9.

5 C. C. Cheng, Y. L. Lo, B. S. Pun, Y. M. Chang and W. Y. Li: J. Lightwave Technol. 23 (2005) 3907.

6 W. Y. Li, C. C. Cheng and Y. L. Lo: Sens. Actuators, A 149 (2009) 201.

7 S. C. Her and C. Y. Tsai: Adv. Mater. Res. 201-203 (2011) 2419.

8 J. S. Sirkis: Opt. Eng. 32 (1993) 752.

9 G. B. Hocker: Appl. Opt. 18 (1979) 1445. 